\title{
Characteristics and Outcomes of Acetaminophen Overdose and Hepatotoxicity in Thailand
}

\author{
Natthiya Pholmoo and Chalermrat Bunchorntavakul* \\ Division of Gastroenterology and Hepatology, Department of Medicine, Rajavithi Hospital, Bangkok, Thailand
}

\begin{abstract}
Background and Aims: Acetaminophen (APAP) is the leading cause of drug overdose and hepatotoxicity worldwide, including in Thailand. Patterns of overdose and hospital management are known to have significant impacts on the outcomes of APAP overdose, and these factors vary from country to country. Therefore, this study aimed to analyze clinical characteristics of Thai patients with APAP overdose in terms of overdose patterns, clinical presentation, treatment and outcomes. Methods: In this retrospective analytical study, medical records of adult patients hospitalized with a diagnosis of APAP overdose at Rajavithi Hospital, Bangkok, between January 2013 and December 2017 were reviewed. Results: A total of 184 patients diagnosed with APAP overdose were included. The median age was $22(15-76)$ years and the majority were female $(79.9 \%)$. Most overdoses were intended self-poisoning ingestion $(90.8 \%)$ with a median dose of $10.5 \mathrm{~g}(4.5-50)$. A total of 121 patients were treated with $\mathrm{N}$-acetylcysteine with a median visit-to- $\mathrm{N}$-acetylcysteine time of $2(0.5-15) \mathrm{h}$. Overall, $15.6 \%$ developed mild hepatotoxicity (aspartate aminotransferase or alanine aminotransferase $>3$ times the upper limit of normal), $6.4 \%$ developed severe hepatotoxicity (aspartate aminotransferase or alanine aminotransferase $>10$ times the upper limit of normal and international normalized ratio $>2.0$ ) and 3 patients developed acute liver failure ( $1 \mathrm{pa}-$ tient resolved spontaneously and 2 patients, neither of whom had a liver transplant, died). Significant predictors for hepatotoxicity included older age, chronic alcohol drinking, repeated taking of medication for more than $8 \mathrm{~h}$ (staggered ingestion), long duration between ingestion and hospital visit, alcohol coingestion, abdominal pain symptoms, and acute kidney injury. Conclusions: Most cases of APAP overdose in Thailand appear to be young women with intentional ingestion. With prompt management, most patients $(76.4 \%)$ did not develop significant hepatotoxicity; nevertheless, despite $\mathrm{N}$-acetylcysteine therapy, hepatotoxicity including acute liver failure was observed in a small proportion of patients, particularly those with unintentional overdose and chronic alcohol drinking.
\end{abstract}

Keywords: Acetaminophen; Paracetamol; Overdose; Drug-induced hepatotoxicity; Liver failure.

Abbreviations: AKI, acute kidney injury; ALF, acute liver failure; APAP, acetaminophen; ALT, alanine aminotransferase; $\mathrm{AST}$, aspartate aminotransferase; $\mathrm{Cr}$, creatinine; CYP, cytochrome; INR, international normalized ratio; NAC, $\mathrm{N}$-acetylcysteine; PT, prothrombin time; RUCAM, Roussel Uclaf Causality Assessment Method; TB, total bilirubin; xULN, times higher than the upper limit of normal.

Received: 19 December 2018; Revised: 15 May 2019; Accepted: 16 May 2019

*Correspondence to: Chalermrat Bunchorntavakul, Division of Gastroenterology and Hepatology, Department of Internal Medicine, Rajavithi Hospital, College of Medicine, Rangsit University, Rajavithi Road, Ratchathewi, Bangkok 10400, Thailand. Tel: +662-3548081, Fax: +662-3548179, E-mail: dr.chalermrat@gmail.com
Citation of this article: Pholmoo N, Bunchorntavakul C. Characteristics and outcomes of acetaminophen overdose and hepatotoxicity in Thailand. J Clin Transl Hepatol 2019;7 (2):132-139. doi: 10.14218/JCTH.2018.00066.

\section{Introduction}

Acetaminophen (APAP) is an over-the-counter medicine which is commonly used worldwide, either as a singleingredient medication or as a component of numerous combination products for analgesia and antipyresis. In Thailand, APAPs are widely available as over-the-counter drugs, which can be sold without restriction in terms of quantities and the number of tablets per bottle. Although APAP is generally considered to be safe at the usual therapeutic doses recommended by the manufacturer ( $1-4 \mathrm{~g} /$ day), concerns about its use have emerged over the past decade as APAP has been increasingly recognized as a major cause of acute liver failure (ALF) in adults in the United States and many other countries worldwide. ${ }^{1,2}$ In contrast, Asia-Pacific countries have a higher incidence of ALF, due to hepatitis viruses and drugs, with fewer cases of APAP overdose being observed. ${ }^{3}$

According to a recent systematic review, about one quarter of patients with drug-induced liver injury in China are associated with traditional Chinese medicine. ${ }^{4}$ Notably, APAP is a classic cause of drug-induced hepatotoxicity in a dose-dependent manner. Single-overdose ingestion typically occurs in attempted suicide, and doses exceeding 15-25 g may cause severe liver injury, resulting in death in up to $25 \%$ of cases. However, it should be noted that $30-50 \%$ of cases of hospitalized APAP hepatotoxicity nowadays result from "unintentional overdose" or a "therapeutic misadventure", whereby the daily dose may not have greatly exceeded the recommended safe limits but where certain risk factors are present, such as concomitant alcohol use, obesity, malnutrition state, and medications that interact with the cytochrome (CYP) system. ${ }^{3,5}$

Patterns of overdose and hospital management are known to have significant impacts on the outcomes of APAP overdose, and these factors vary between countries, but in Southeast Asia the data on APAP overdose and hepatotoxicity are limited. Previous studies from Malaysia and Singapore have reported somewhat different characteristics and better outcomes in patients with APAP overdose compared to those of studies from Western countries. ${ }^{6-10}$ Therefore, this study aimed to describe the clinical characteristics of Thai patients with APAP overdose in terms of overdose patterns, clinical presentation, treatment, and outcomes. In addition, the features and predictive factors of APAP-induced hepatotoxicity were also analyzed. 


\section{Methods}

\section{Study oversight}

Medical records of consecutive adult patients hospitalized with diagnosis of APAP overdose at Rajavithi Hospital, Bangkok over a five-year period from January 2013 to December 2017 were retrospectively reviewed, and the results were matched with the International Statistical Classification of Disease and Related Health Problems, $10^{\text {th }}$ revision T39.1 (poisoning by non-opioid analgesics, antipyretics, and antirheumatics [4-aminophenol derivatives]). The study protocol was reviewed and approved by the Medical Ethics Committee of Rajavithi Hospital.

\section{Study population}

Adult patients ( $>15$ years of age) who presented at the Emergency Department of the hospital with a diagnosis of APAP overdose were consecutively included. Patients with incomplete or unclear records were excluded. Patient demographics were reviewed and recorded as follows: age, gender, length of stay, cost, comorbid diseases, history of alcohol intake, dose and pattern of ingestion, time to hospital visit, coingestants, clinical presentations, physical examination, laboratory data (e.g., aspartate aminotransferase (AST), alanine aminotransferase (ALT), total bilirubin (TB), prothrombin time (PT), international normalized ratio (INR), creatinine $(\mathrm{Cr})$, platelet count, hematocrit), treatments, and clinical outcomes. Intentionality of overdose was also noted and compared.

\section{Term definitions}

APAP overdose was defined as an ingestion of supratherapeutic dose of $>4 \mathrm{~g}$ per day, $>2 \mathrm{~g}$ per day for alcoholic patients, or levels higher than therapeutic ones ( $>20 \mathrm{mcg} / \mathrm{L}$ ) for patients with chronic liver disease. Acute overdose was defined as an ingestion of APAP overdose within an 8-h period. Supratherapeutic doses ingested over a time period of over $8 \mathrm{~h}$ were considered as a staggered overdose or a repeated supratherapeutic ingestion, which is referred to as a "staggered ingestion" in this study. Unintentional overdose was defined as an ingestion of supratherapeutic dose for pain/ fever reduction without the intention of self-harm. Mild hepatotoxicity was defined as present when either the AST or ALT reading was 3 times higher than the upper limit of normal (xULN) but not more than 10 xULN.

Severe hepatotoxicity was defined as present when either the AST or ALT reading was $10 x U L N$ with an INR $>2 .{ }^{11}$ ALF was defined as hepatotoxicity with an INR $>1.5$ with any degree of hepatic encephalopathy. ${ }^{12}$ Acute kidney injury (AKI) was defined as an absolute increase in serum $\mathrm{Cr}$ of $0.3 \mathrm{mg} / \mathrm{dL}$ or a percentage increase in serum $\mathrm{Cr}$ of $50 \%$ within $48 \mathrm{~h}^{13}$

\section{Statistical analysis}

All results were analyzed using the Statistical Package for Social Scientists (SPSS version 23.0). Data values were presented as median and range (minimum to maximum). Demographic data and baseline characteristics were summarized using descriptive statistics such as mean \pm standard deviation or median (min-max). Categorical variables were compared using the chi-square test or Fisher's exact test, while comparison of continuous variables was performed using the independent $t$-test or the Mann-Whitney $U$ test. Logistic regression analysis was used to analyze the significant predictors of hepatotoxicity and severe hepatotoxicity, and results are presented by odds ratio. All statistical examinations were two-tailed with $p$-value $<0.05$ defined as statistically significant.

\section{Results}

\section{Baseline demographic data and patterns of ingestion}

A total of 184 patients with APAP overdose were included (17 unintentional and 167 intentional overdose ingestions). The median age was 22 (15-76) years, and $79.9 \%$ were female. Among the 57 patients whose complete history of alcohol consumption was obtained, $15.8 \%$ were chronic alcohol drinkers. The median amount of APAP ingestion was 10.5 $(4.5-50) \mathrm{g}$. Alcohol coingestion was reported in 10 patients (5.4\%) and drug/substance coingestion was reported in 28 $(15.2 \%)$ patients (14 antihistamines, 7 nonsteroidal antiinflammatory drugs, 2 sleeping pills, 2 antibiotics, 1 antacid, 1 unknown drug, and 2 toilet cleaning liquids). Comparison of the two groups showed that patients with unintentional overdose were more likely to be male, older, and have history of alcohol abuse, underlying cirrhosis, longer duration of APAP ingestions (staggered ingestion), and longer time between ingestion and hospital visit (Table 1 ).

\section{Clinical presentation and laboratory findings}

The common symptoms reported at presentation were nausea/vomiting (66.8\%) and abdominal pain $(31.5 \%)$. Five patients, all of whom were cases of unintentional overdose, had jaundice. Laboratory data are summarized in Table 2. Most patients had serum AST, ALT, TB, Cr, PT, INR and complete blood count within the normal ranges throughout hospitalization. In those with documented hepatotoxicity, the peak AST and ALT levels were 15,616 IU/L and 7,726 IU/L respectively, with median duration of $10-18$ (1-105) h after acute overdose. Eleven patients $(6 \%)$ had AKI, with maximum $\mathrm{Cr}$ of $5.37 \mathrm{mg} / \mathrm{dL}$. Comparison of the two groups showed that patients with unintentional overdose were more likely to have jaundice, hepatotoxicity and AKI, whereas those with intentional overdose were likely to have nausea and vomiting at presentation.

\section{Hospital management and clinical outcomes}

Patients with intentional APAP overdose underwent gastric lavage and activated charcoal administration, involving $70(41 \%)$ and $71(42.5 \%)$ cases respectively. Most patients $(65.8 \%)$ had average interval from ingestion to $\mathrm{N}$-acetylcysteine (NAC) administration of $10(1-337) \mathrm{h}$ and were treated with intravenous NAC. The average interval from hospital arrival to NAC administration was $2(0.5-15)$ h. Serum for APAP concentration was obtained from 121 of 128 patients (94\%) who presented $<24$ hours after ingestion. Fifty-nine patients had serum APAP concentration above the "treatment line" of the Rumack-Matthew Nomogram, and fifty-eight of these (98\%) received NAC. Sixty-two patients had serum APAP concentration below the treatment line, and twenty-one of them (34\%) received NAC (Fig. 1 ). 


\begin{tabular}{|c|c|c|c|c|}
\hline & All, $n=184$ & Unintentional, $n=17$ & Intentional, $n=167$ & $p$-value \\
\hline \multicolumn{5}{|l|}{ Variable } \\
\hline Age in years & $22[15,76]$ & $33[15,50]$ & $21[15,76]$ & $<0.001$ \\
\hline Female gender & $147(79.9 \%)$ & $11(64.7 \%)$ & $136(81.4 \%)$ & 0.115 \\
\hline Length of stay in days & $2[1,21]$ & $5[1,21]$ & $2[1,14]$ & 0.014 \\
\hline \multicolumn{5}{|l|}{ Comorbid disease } \\
\hline None & $177(96.2 \%)$ & $14(82.4 \%)$ & $163(97.6 \%)$ & 0.018 \\
\hline Cirrhosis & $2(1.1 \%)$ & $2(11.8 \%)$ & $0(0 \%)$ & 0.008 \\
\hline Psychiatric disease & $3(1.6 \%)$ & $0(0 \%)$ & $3(1.8 \%)$ & 1.000 \\
\hline \multicolumn{5}{|l|}{ History of alcohol use* } \\
\hline Chronic drinker & $9 / 57(15.8 \%)$ & $5 / 11(45.4 \%)$ & $4 / 46(8.7 \%)$ & $<0.001$ \\
\hline Social drinker & $17 / 57(29.8 \%)$ & $4 / 11(36.4 \%)$ & $13 / 46(28.3 \%)$ & 0.056 \\
\hline None/rare & $31 / 57(54.4 \%)$ & $2 / 11(18.2 \%)$ & $29 / 46(63.0 \%)$ & 0.742 \\
\hline \multicolumn{5}{|l|}{ Time of ingestion } \\
\hline Acute $(<8 \mathrm{~h})$ & $170(92.4 \%)$ & $4(23.5 \%)$ & $166(99.4 \%)$ & $<0.001$ \\
\hline Staggered (> $8 \mathrm{~h})$ & $11(6 \%)$ & $11(64.7 \%)$ & $0(0 \%)$ & $<0.001$ \\
\hline Unknown & $3(1.6 \%)$ & $2(11.8 \%)$ & $1(0.6 \%)$ & 0.023 \\
\hline Time to hospital in $\mathbf{h}$ & $6[1,1008]$ & $72[1,1008]$ & $6[1,84]$ & $<0.001$ \\
\hline Ingested dose in $\mathbf{g}$ & $10.5[4.5,50]$ & $12[4.5,30]$ & $10.5[4.5,50]$ & 0.332 \\
\hline Coingestants & $36(19.6 \%)$ & $2(11.8 \%)$ & $34(20.4 \%)$ & 0.532 \\
\hline Alcohol & $10(5.4 \%)$ & $2(11.8 \%)$ & $8(4.8 \%)$ & 0.233 \\
\hline Other drug(s) & $28(15.2 \%)$ & $0(0 \%)$ & $28(16.8 \%)$ & 0.080 \\
\hline
\end{tabular}

Values are presented as number (\%) and median [range].

*missing data $69 \%$.

In terms of clinical outcomes, 132 of the 173 patients $(76.3 \%)$ had no significant hepatotoxicity on admission. Overall, 27 patients $(15.6 \%)$ developed mild hepatotoxicity, $11(6.4 \%)$ developed severe hepatotoxicity, and $3(1.7 \%)$ developed ALF (1 spontaneously resolved, and 2 deaths without liver transplant). Treatment outcome was unable to be documented in 11 patients in the intentional overdose group, due to entering and leaving the hospital within a very short period of time for personal reasons (e.g., refusing treatment or changing hospital). Patients with unintentional overdose were more likely to develop hepatotoxicity and ALF than those who had intentionally overdosed (Table 3). Among the 121 patients whose serum APAP concentration was documented, all patients who developed hepatotoxicity had serum APAP concentration above the treatment line of the RumackMatthew Nomogram (Fig. 1).

\section{Predictive factors for APAP-induced hepatotoxicity}

Based on univariate logistic regression analysis, significant predictors of APAP-induced hepatotoxicity included older age, male gender, chronic alcohol drinking, unintentional overdose, staggered ingestion, alcohol coingestion, late presentation, and presence of abdominal pain. Patients with hepatotoxicity more frequently developed AKI (17.1\% vs. $3 \%$ respectively) and required longer hospital stay ( 5 days vs. 1 day respectively) compared to those without it (Table 4). Significant predictors of severe hepatotoxicity included older age, chronic alcohol drinking, underlying cirrhosis, unintentional overdose, staggered ingestion, alcohol coingestion, late presentation, and presence of abdominal pain and jaundice. Patients with hepatotoxicity more frequently developed AKI ( $42.9 \%$ vs. $3.1 \%$ respectively) and required longer hospital stay ( 7.5 days vs. 2 days, respectively) than those without it (Table 5). Multivariate analysis did not identify any significant independent predictors of hepatotoxicity, whereas the presence of abdominal pain was an independent predictor of severe hepatotoxicity (adjusted odds ratio: $12.61,95 \%$ confidence interval: $1.91-83.27 ; p=0.009$ ).

\section{Discussion}

Our study comprehensively depicted the patient characteristics, patterns, and outcomes of APAP overdose in Thailand. Rajavithi Hospital is a referral hospital under the Ministry of Public Health of Thailand, located in central Bangkok, and the authors believe that the clinical characteristics of patients included in this study can be a good representation of APAP overdose patients who present at secondary/tertiary medical centers in Bangkok. Similar to the findings of previous reports from Asia-Pacific regions (e.g., Singapore, ${ }^{10}$ Malaysia, ${ }^{9}$ and Australia) ${ }^{14}$ the majority of APAP overdose cases were female ( $80 \%)$ with an average age of $22-25$ years, and up to $90 \%$ of cases were deliberate overdose with the intent of self-harm. Histories of chronic alcohol drinking, substance coingestion, and significant medical comorbidities were relatively uncommon in this study, and this is in keeping with the results of other reports from the Asia-Pacific region. $9,10,14$ 
Pholmoo N. et al: Acetaminophen hepatotoxicity in Thailand

Table 2. Clinical presentation, physical examination and laboratory data

\begin{tabular}{|c|c|c|c|c|}
\hline & All, $n=184$ & Unintentional, $n=17$ & Intentional, $n=167$ & $p$-value \\
\hline \multicolumn{5}{|l|}{ Clinical presentation } \\
\hline Asymptomatic & $36(19.6 \%)$ & $5(29.4 \%)$ & $31(18.6 \%)$ & 0.283 \\
\hline Nausea/vomiting & $123(66.8 \%)$ & $8(47.1 \%)$ & $115(68.9 \%)$ & 0.102 \\
\hline Abdominal pain & $58(31.5 \%)$ & $6(35.3 \%)$ & $52(31.1 \%)$ & 0.786 \\
\hline Dizziness & $16(8.7 \%)$ & $0(0 \%)$ & $16(9.6 \%)$ & 0.369 \\
\hline Drowsiness & $10(5.4 \%)$ & $2(11.8 \%)$ & $8(4.8 \%)$ & 0.233 \\
\hline \multicolumn{5}{|l|}{ Physical examination } \\
\hline Normal & $120(65.2 \%)$ & $6(35.3 \%)$ & $114(68.3 \%)$ & 0.007 \\
\hline Jaundice & $5(2.7 \%)$ & $5(29.4 \%)$ & $0(0 \%)$ & $<0.001$ \\
\hline Hepatomegaly & $1(0.5 \%)$ & $1(5.9 \%)$ & $0(0 \%)$ & 0.092 \\
\hline Abdominal pain & $59(32.1 \%)$ & $6(35.3 \%)$ & $53(31.7 \%)$ & 0.788 \\
\hline Encephalopathy & $1(0.5 \%)$ & $1(5.9 \%)$ & $0(0 \%)$ & 0.092 \\
\hline \multicolumn{5}{|l|}{ Laboratory findings } \\
\hline Peak AST as U/L & $24[12,15616]$ & $899[12,15616]$ & $23[12,8615]$ & 0.001 \\
\hline Time in $\mathrm{h}$ & $10.5[1,1018]$ & $78[1,1018]$ & $10[1,88]$ & 0.001 \\
\hline Peak ALT as U/L & $19[6,7726]$ & $583[9,4945]$ & $18[6,7726]$ & 0.003 \\
\hline Time in $\mathrm{h}$ & $10.5[1,1018]$ & $78[1,1018]$ & $10[1,105]$ & 0.001 \\
\hline Peak INR & $1.13[0.8,6.14]$ & $1.79[1.02,5.24]$ & $1.12[0.8,6.14]$ & 0.001 \\
\hline Time in $\mathrm{h}$ & $13[1,1009]$ & $85[1,1009]$ & $12[1,105]$ & 0.001 \\
\hline Peak TB as $\mathrm{mg} / \mathrm{dL}$ & $0.76[0.1,37.7]$ & $4.87[0.25,37.7]$ & $0.74[0.1,12.28]$ & 0.004 \\
\hline Time in $\mathrm{h}$ & $14.5[1,1009]$ & $117[1,1009]$ & $13.5[1,134]$ & 0.003 \\
\hline Peak creatinine as $\mathrm{mg} / \mathrm{dL}$ & $0.64[0.27,5.37]$ & $0.93[0.34,5.37]$ & $0.63[0.27,4]$ & 0.013 \\
\hline Time in $\mathrm{h}$ & $8[1,1009]$ & $90.5[1,1009]$ & $8[1,129]$ & 0.001 \\
\hline Platelet as /uL) & $286000[16000,523000]$ & $204000[74000,363000]$ & $288000[16000,523000]$ & 0.005 \\
\hline Hematocrit as $\%$ & $38.8[25.6,53.1]$ & $39[31.1,50.6]$ & $38.8[25.6,53.1]$ & 0.958 \\
\hline Acute kidney injury & $11(6 \%)$ & $6(35.3 \%)$ & $5(3 \%)$ & $<0.001$ \\
\hline
\end{tabular}

Values are presented as number (\%) and median [range].

Abbreviations: AST, aspartate aminotransferase; ALT, alanine aminotransferase; TB, total bilirubin; INR, international normalized ratio.

Notably, patient characteristics and patterns of acute overdose in this study are somewhat different from those reported from Western countries. In the USA, national surveillance systems assessed rates of APAP-related events identified in different settings, including calls to poison centers, emergency department visits, and in-patient hospitalizations, and estimated that the prevalence of female gender with APAP overdose was $62-69 \%$, and that $16-67 \%$ of cases were unintentional overdoses. ${ }^{15} \mathrm{~A}$ higher prevalence of unintentional APAP overdose among all cases has also been observed in European countries. ${ }^{16}$ In addition, substance coingestion seems to be more common in Western countries than in Asia-Pacific ones. ${ }^{15,16}$

On presentation, abdominal symptoms, most commonly nausea and vomiting, were reported in more than $60 \%$ of cases. Interestingly, abdominal pain corresponding with mild abdominal tenderness, either at the epigastric (most cases) or right upper quadrant area, was observed in about $32 \%$ of our patients with acute overdose and appeared to be associated with the subsequent development of APAP-induced hepatotoxicity (2.4-fold increased risk); to the best of our knowledge, this observation has never been previously reported elsewhere. The exact explanation is unclear but abdominal pain may be related to gastric irritation or inflammation induced by APAP exposure at higher doses, or, in rare instances, may be related to distension of the liver capsule associated with hepatocyte injury. Although this observation needs to be confirmed in future studies, the presence of abdominal pain could be another simple clinical predictor of APAP-induced hepatotoxicity, particularly when the ingested dose is uncertain and serum APAP is not readily available.

Of patients with intentional overdose, only $14.1 \%$ and $5.1 \%$ developed mild or severe hepatotoxicity respectively. There was no incidence of ALF or death. Notably, 8 patients who developed severe hepatotoxicity from intentional overdose came to the hospital late after ingestion, and only 2 received NAC therapy within $24 \mathrm{~h}$. The good outcomes in this group of patients may be due to: (1) patient characteristics, including young age, lack of underlying liver disease, and early presentation to the hospital (median time $6 \mathrm{~h}$ from ingestion); and (2) hospital management, particularly prompt NAC therapy (median time of not $>2 \mathrm{~h}$ after presentation). In cases of unintentional overdose, $35.3 \%, 29.4 \%$, and $17.6 \%$ of patients developed mild hepatotoxicity, ALF, or died respectively. The poorer outcomes in this group may be 
Pholmoo N. et al: Acetaminophen hepatotoxicity in Thailand

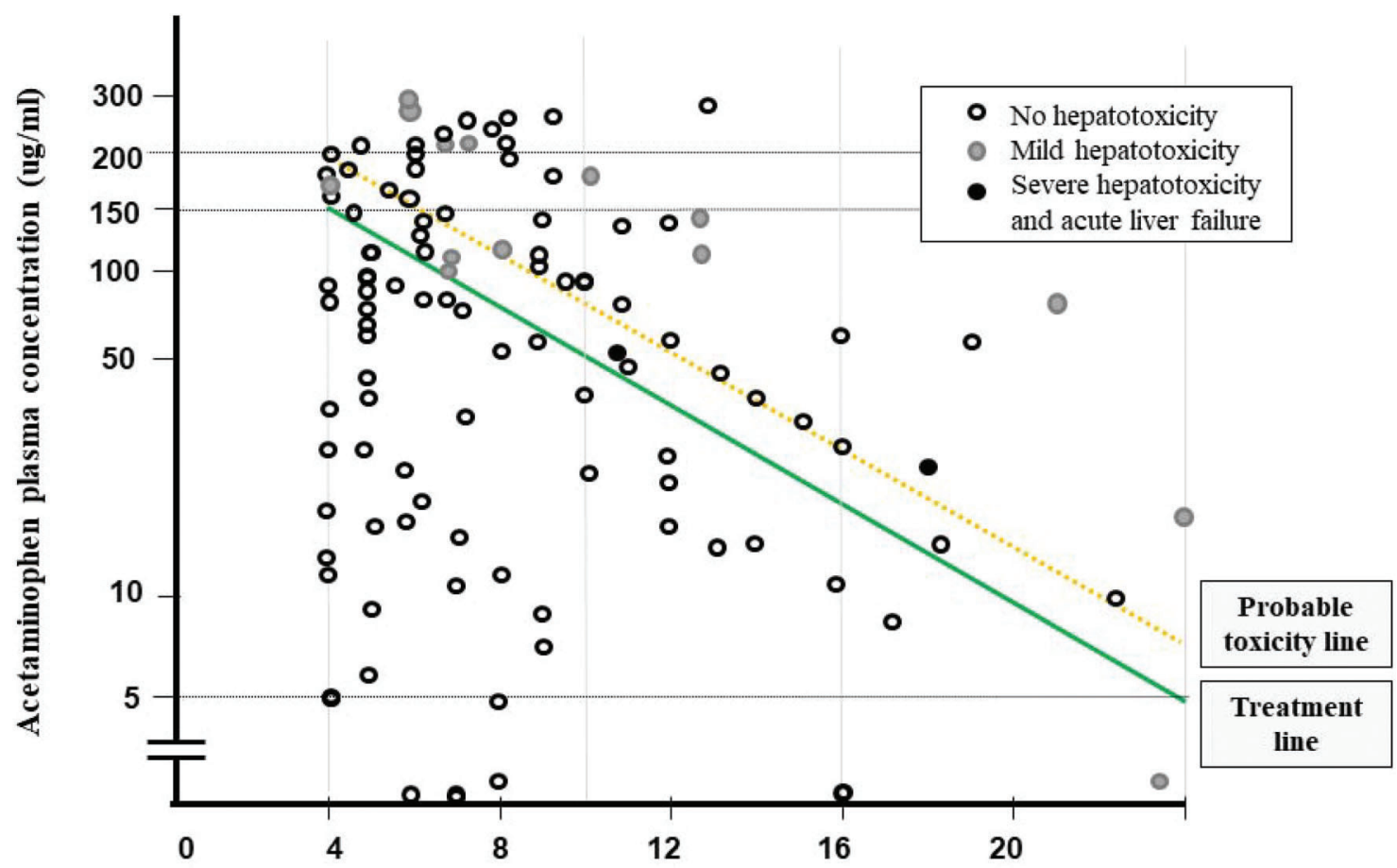

Hours after ingestion

Fig. 1. Individual value plot: acetaminophen concentration versus time after single acute overdose. Sloped lines are the "probable toxicity line" and the "treatment line" according to the Rumack-Matthew nomogram and corresponding to 200 and $500 \mu \mathrm{g} / \mathrm{mL}$ at $4 \mathrm{~h}$ post-ingestion respectively.

mainly due to comorbidities and late presentation to the hospital (many of the patients came to the hospital when evident hepatotoxicity had already developed).

The differences between the characteristics of patients with intentional versus unintentional APAP overdose were similar to those in previous reports. ${ }^{5,8,17}$ It should be noted that the overall incidence of severe APAP-induced hepatotoxicity among patients with APAP overdose in our cohort $(6.4 \%)$ was quite low compared to other reports worldwide: $31 \%$ in UK $(n=80)^{6} ; 32 \%$ in Texas, USA $(n=71)^{8} ; 15 \%$ in multi-states,

Table 3. Hospital management and outcomes

\begin{tabular}{|c|c|c|c|c|}
\hline & All, $n=184$ & Unintentional, $n=17$ & Intentional, $n=167$ & $p$-value \\
\hline \multicolumn{5}{|l|}{ Treatment } \\
\hline Gastric lavage & $70(38 \%)$ & $0(0 \%)$ & $70(41.9 \%)$ & $<0.001$ \\
\hline Activated charcoal & $71(38.6 \%)$ & $0(0 \%)$ & $71(42.5 \%)$ & $<0.001$ \\
\hline Intravenous NAC & $121(65.8 \%)$ & $12(70.6 \%)$ & $109(65.3 \%)$ & 0.792 \\
\hline Ingestion-to-NAC time in $\mathrm{h}$ & $10[1,337]$ & $78[9,337]$ & $9[1,93]$ & $<0.001$ \\
\hline Visit-to-NAC time in $\mathrm{h}$ & $2[0.5,15]$ & $4[0.5,15]$ & $2[0.5,11]$ & 0.034 \\
\hline Acetaminophen level as $\mathrm{mcg} / \mathrm{mL}$ & $50.3[0,335.3]$ & $17.8[0,218.3]$ & $57.65[0,335.3]$ & 0.002 \\
\hline Time in $\mathrm{h}$ & $7[1,1018]$ & $76.5[1,1018]$ & $7[1,87]$ & $<0.001$ \\
\hline \multicolumn{5}{|l|}{ Outcome } \\
\hline No hepatotoxic injury & $132 / 173(76.3 \%)$ & $6 / 17(35.3 \%)$ & $126 / 156(80.8 \%)$ & 0.001 \\
\hline Mild hepatotoxic injury & $27 / 173(15.6 \%)$ & $5 / 17(29.4 \%)$ & $22 / 156(14.1 \%)$ & 0.140 \\
\hline Severe hepatotoxic injury & $11 / 173(6.4 \%)$ & $3 / 17(17.6 \%)$ & $8 / 156(5.1 \%)$ & 0.068 \\
\hline Acute liver failure & $3 / 173(1.7 \%)$ & $3 / 17(17.6 \%)$ & $0 / 156(0 \%)$ & 0.001 \\
\hline Death & $2 / 173(1.2 \%)$ & $2 / 17(11.8 \%)$ & $0 / 156(0 \%)$ & 0.008 \\
\hline
\end{tabular}

Values presented as number (\%) and median [range].

Abbreviation: NAC, $\mathrm{N}$-acetylcysteine. 
Pholmoo N. et al: Acetaminophen hepatotoxicity in Thailand

Table 4. Univariate logistic regression analysis: predictive factors of APAP-induced hepatotoxicity

\begin{tabular}{|c|c|c|c|c|}
\hline & No hepatotoxicity, $n=132$ & Hepatotoxicity, $n=41$ & OR $(95 \% \mathrm{CI})$ & $p$-value \\
\hline Age in years & $21[15,76]$ & $26[16,50]$ & $1.05(1.01,1.09)$ & 0.007 \\
\hline Female gender & $110(83.3 \%)$ & $27(65.9 \%)$ & $0.39(0.18,0.85)$ & 0.018 \\
\hline Chronic alcohol drinker & $1(0.8 \%)$ & $8(19.5 \%)$ & $4.42(3.01,6.48)$ & $<0.001 *$ \\
\hline Underlying cirrhosis & $0(0 \%)$ & $2(4.9 \%)$ & $4.38(3.33,5.78)$ & 0.055 \\
\hline Intentional overdose & $126(95.5 \%)$ & $30(73.2 \%)$ & $0.3(0.18,0.48)$ & $<0.001 *$ \\
\hline Unintentional overdose & $6(4.5 \%)$ & $11(26.8 \%)$ & $3.36(2.09,5.42)$ & $<0.001^{*}$ \\
\hline Acute ingestion & $129(97.7 \%)$ & $30(73.2 \%)$ & $0.24(0.16,0.37)$ & $<0.001 *$ \\
\hline Staggered ingestion & $3(2.3 \%)$ & $8(19.5 \%)$ & $3.57(2.22,5.73)$ & $0.001 *$ \\
\hline Ingested dose in $\mathrm{g}$ & $10[4.5,50]$ & $15[4.5,50]$ & $1.03(1,1.06)$ & 0.079 \\
\hline Time to hospital in $\mathrm{h}$ & $5[1,96]$ & $37[1,1008]$ & $1.05(1.03,1.07)$ & $<0.001^{*}$ \\
\hline Alcohol coingestion & $4(3 \%)$ & $5(12.2 \%)$ & $4.44(1.13,17.42)$ & $0.032 *$ \\
\hline Abdominal pain & $38(28.8 \%)$ & $20(48.8 \%)$ & $2.36(1.15,4.84)$ & 0.019* \\
\hline Acute kidney injury & $4(3 \%)$ & $7(17.1 \%)$ & $6.63(1.83,24.01)$ & $0.004 *$ \\
\hline Gastric lavage & $60(45.5 \%)$ & $6(14.6 \%)$ & $0.21(0.08,0.52)$ & $0.001 *$ \\
\hline Activated charcoal & $62(47 \%)$ & $6(14.6 \%)$ & $0.19(0.08,0.49)$ & $0.001 *$ \\
\hline Intravenous NAC & $82(62.1 \%)$ & $38(92.7 \%)$ & $7.72(2.26,26.34)$ & $0.001 *$ \\
\hline Time from ingestion to NAC & $8.5[1,100]$ & $40[6,337]$ & $1.08(1.04,1.11)$ & $<0.001^{*}$ \\
\hline Length of stay in days & $1[1,5]$ & $5[1,21]$ & $2.57(1.84,3.57)$ & $<0.001 *$ \\
\hline
\end{tabular}

Values are presented as number (\%) and median [range].

Abbreviations: $\mathrm{CI}$, confidence interval; NAC, N-acetylcysteine; OR, odds ratio.

USA $(n=157)^{7} ; 14 \%$ in Australia $(n=188)^{18} ; 7.3 \%$ in Malaysia $(n=1024)^{9} ; 5.6 \%$ in Singapore $(n=177)^{10}$; and $6 \%$ in Hong Kong $(n=104) .{ }^{19}$ Although the definition of severe hepatotoxicity may vary in different studies, it appears that the incidence of APAP-induced hepatotoxicity among patients with APAP overdose is lower in Asia than in Western countries. One possible explanation for this observation is the difference in the total dose of APAP ingestion, mostly with intent

Table 5. Univariate logistic regression analysis: predictive factors of APAP-induced severe hepatotoxicity

\begin{tabular}{|c|c|c|c|c|}
\hline & $\begin{array}{l}\text { No severe hepatotoxicity, } \\
* n=159\end{array}$ & $\begin{array}{l}\text { Severe hepatotoxicity, } \\
* * n=14\end{array}$ & OR $(95 \% \mathrm{CI})$ & p-value \\
\hline Age in years & $21[15,76]$ & $33.5[18,50]$ & $1.08(1.03,1.13)$ & $0.001 *$ \\
\hline Female gender & $131(78.9 \%)$ & $11(78.6 \%)$ & $0.98(0.24,5.76)$ & 1.0 \\
\hline Chronic alcohol drinker & $2(1.3 \%)$ & $7(50 \%)$ & $18.22(8.15,40.74)$ & $<0.001 *$ \\
\hline Underlying cirrhosis & $0(0 \%)$ & $2(14.3 \%)$ & $14.25(8.26,24.59)$ & $0.006 *$ \\
\hline Intentional overdose & $148(93.1 \%)$ & $8(57.1 \%)$ & $0.15(0.06,0.37)$ & $0.001 *$ \\
\hline Unintentional overdose & $11(6.9 \%)$ & $6(42.9 \%)$ & $6.88(2.71,17.49)$ & $0.001 *$ \\
\hline Acute ingestion & $151(95 \%)$ & $8(57.1 \%)$ & $0.12(0.05,0.29)$ & $<0.001 *$ \\
\hline Staggered ingestion & $7(4.4 \%)$ & $4(28.6 \%)$ & $5.89(2.2,15.79)$ & $0.007^{*}$ \\
\hline Alcohol coingestion & $6(3.8 \%)$ & $3(21.4 \%)$ & $6.95(1.53,31.64)$ & $0.012 *$ \\
\hline Abdominal pain & $49(30.8 \%)$ & $9(64.3 \%)$ & $4.04(1.29,12.68)$ & $0.017 *$ \\
\hline Acute kidney injury & $5(3.1 \%)$ & $6(42.9 \%)$ & $22.5(5.64,89.76)$ & $<0.001 *$ \\
\hline Gastric lavage & $62(39 \%)$ & $4(28.6 \%)$ & $0.63(0.19,2.08)$ & 0.445 \\
\hline Activated charcoal & $65(40.9 \%)$ & $3(21.4 \%)$ & $0.39(0.11,1.47)$ & 0.166 \\
\hline Intravenous NAC & $106(66.7 \%)$ & $14(100 \%)$ & NA & 0.997 \\
\hline Time from ingestion to NAC & $9[1,337]$ & $56[11,111]$ & $1.02(1,1.03)$ & $0.034 *$ \\
\hline Length of stay in days & $2[1,21]$ & $7.5[3,14]$ & $1.4(1.2,1.62)$ & $<0.001 *$ \\
\hline
\end{tabular}

Values are presented as number (\%) and median [range].

Abbreviations: $\mathrm{CI}$, confidence interval; NAC, N-acetylcysteine; OR, odds ratio. 
of self-harm, which was higher among studies from the UK and USA (median: $15-18 \mathrm{~g})^{6-8}$ than in studies from AsiaPacific regions (median 10-12 g). ${ }^{9,10,18,19}$

Apart from overdose intention, several other factors have been found to predict the subsequent development of hepatotoxicity, including older age, chronic alcohol drinking, staggered ingestion, long duration between ingestion and hospital visit, alcohol coingestion, abdominal pain symptoms, and AKI. These factors, with the exception of abdominal pain (discussed above), have been found to be associated with APAP-induced hepatotoxicity. ${ }^{2,9,17,20}$ The interaction between ethanol, a competitive substrate of CYP2E1, and APAP is complex. Acute alcohol ingestion is not a risk factor for APAP hepatotoxicity and may actually be protective by competing with APAP for CYP2E1.20,21 Chronic alcohol ingestion potentiates APAPinduced hepatotoxicity by up-regulating CYP2E1 and decreasing glutathione synthesis. Most available data have concluded that chronic alcohol consumption is associated with an increased risk of APAP-induced hepatotoxicity in patients with repeated overdoses (mostly therapeutic misadventure), particularly in those who have underlying cirrhosis; however, alcoholics do not seem to be at an increased risk of hepatotoxicity at a therapeutic dose or in a single overdose setting. 2,21,22 Nonetheless, our study found that both acute and chronic alcohol drinking were risk factors of APAP-induced hepatotoxicity.

According to recent guidelines for the management of APAP poisoning in Australia and New Zealand, APAP concentration should be used to assess the need for NAC administration in all patients presenting with deliberate self-poisoning with APAP, regardless of the stated dose. ${ }^{23}$ In this study, serum was obtained for measuring APAP concentration from $94 \%$ of patients who presented within $24 \mathrm{~h}$ after ingestion and from 58 of 59 patients ( $98 \%$ ) who had APAP concentrations above the treatment line who had received NAC therapy (all intravenously). All patients who developed hepatotoxicity had serum APAP concentration levels above the treatment line, supporting the recommendation of the use of APAP concentration and the Rumack-Matthew Nomogram for NAC treatment justification in Thai populations. Notably, NAC therapy tended to be overutilized in our hospital, as 21 of 62 patients (34\%) who had serum APAP concentration below the treatment line also received NAC. In addition, anaphylactoid reaction was observed in only $2 / 121$ patients $(1.7 \%)$ in this study, which appeared to be much lower than in previous literature (10-20\%). ${ }^{2}$

This study had several limitations, including its retrospective nature, its single-center design and relatively small number of patients, especially in the unintentional overdose group. It is also possible that the total dose of APAP intake estimated by the patients could have been inaccurate, and data on the amount of alcohol consumption was not clearly documented (missing or incomplete) in $69 \%$ of patients. Furthermore, utilization of the Roussel Uclaf Causality Assessment Method (referred to as RUCAM) and biomarkers would have helped to improve the causality assessment in suspected cases of hepatotoxicity from drugs, including APAP. $^{24,25}$ However, the RUCAM scale was not performed routinely for diagnosis of APAP hepatotoxicity in this study. In future cases, the prospective use of the updated RUCAM is recommended in order to harmonize the causality assessment of drug- and herb-induced liver injury. ${ }^{26}$ Despite these limitations, we did our best to complete the data collection and analysis. It should be noted that this retrospective study has detailed data on the clinical progression of patients, particularly in the hepatotoxicity aspect, and we believe that these data provide valuable insights and may be used as a reference in future related research.

In conclusion, most cases of APAP overdose in Thailand appeared to be young women with intentional ingestion. With prompt management, most patients did not develop significant hepatotoxicity. Even so, despite NAC therapy, hepatotoxicity, including ALF, was observed in a small proportion of patients, and clinical predictors included unintentional overdose, staggered ingestion, older age, chronic alcohol drinking, late presentation, and having abdominal pain.

\section{Conflict of interest}

The authors have no conflict of interests related to this publication.

\section{Author contributions}

Data acquisition, analysis and interpretation, drafting and approval of the final manuscript (NP), conceptualization, analysis and interpretation of data, critical revision and approval of the final manuscript (CB).

\section{References}

[1] Lee WM. Acetaminophen and the U.S. Acute Liver Failure Study Group: lowering the risks of hepatic failure. Hepatology 2004;40:6-9. doi: 10 . 1002/hep.20293.

[2] Bunchorntavakul C, Reddy KR. Acetaminophen-related hepatotoxicity. Clin Liver Dis 2013:17:587-607. doi: 10.1016/j.cld.2013.07.005.

[3] Bunchorntavakul C, Reddy KR. Acetaminophen (APAP or N-Acetyl-p-Aminophenol) and acute liver failure. Clin Liver Dis 2018;22:325-346. doi: 10.1016/j.cld. 2018.01.007.

[4] Wang R, Qi X, Yoshida EM, Méndez-Sánchez N, Teschke R, Sun M, et al. Clinical characteristics and outcomes of traditional Chinese medicineinduced liver injury: a systematic review. Expert Rev Gastroenterol Hepatol 2018;12:425-434. doi: 10.1080/17474124.2018.1427581.

[5] Larson AM, Polson J, Fontana RJ, Davern TJ, Lalani E, Hynan LS, et al. Acetaminophen-induced acute liver failure: results of a United States multicenter, prospective study. Hepatology 2005;42:1364-1372. doi: 10.1002/hep. 20948.

[6] Hawton K, Ware C, Mistry H, Hewitt J, Kingsbury S, Roberts D, et al. Paracetamol self-poisoning. Characteristics, prevention and harm reduction. $\mathrm{Br}$ J Psychiatry 1996;168:43-48. doi: 10.1192/bjp.168.1.43.

[7] James LP, Capparelli EV, Simpson PM, Letzig L, Roberts D, Hinson JA, et al. Acetaminophen-associated hepatic injury: evaluation of acetaminophen protein adducts in children and adolescents with acetaminophen overdose. Clin Pharmacol Ther 2008;84:684-690. doi: 10.1038/clpt.2008.190.

[8] Schiødt FV, Rochling FA, Casey DL, Lee WM. Acetaminophen toxicity in an urban county hospital. N Engl J Med 1997;337:1112-1117. doi: 10. 1056/NEJM199710163371602.

[9] Marzilawati AR, Ngau YY, Mahadeva S. Low rates of hepatotoxicity among Asian patients with paracetamol overdose: a review of 1024 cases. BMC Pharmacol Toxicol 2012;13:8. doi: 10.1186/2050-6511-13-8.

[10] Tan CJ, Sklar GE. Characterisation and outcomes of adult patients with paracetamol overdose presenting to a tertiary hospital in Singapore. Singapore Med J 2017;58:695-702. doi: 10.11622/smedj.2016170.

[11] Koch DG, Speiser JL, Durkalski V, Fontana RJ, Davern T, McGuire B, et al. The natural history of severe acute liver injury. Am J Gastroenterol 2017;112: 1389-1396. doi: 10.1038/ajg.2017.98.

[12] Wendon J, Cordoba J, Dhawan A, Larsen FS, Manns M, Samuel D, et al. EASL Clinical Practical Guidelines on the management of acute (fulminant) liver failure. J Hepatol 2017;66:1047-1081. doi: 10.1016/j.jhep.2016.12.003.

[13] Khwaja A. KDIGO clinical practice guidelines for acute kidney injury. Nephron Clin Pract 2012;120:c179-c184. doi: 10.1159/000339789.

[14] Singer AJ, Carracio TR, Mofenson HC. The temporal profile of increased transaminase levels in patients with acetaminophen-induced liver dysfunction. Ann Emerg Med 1995;26:49-53. doi: 10.1016/S0196-0644(95) 70237-7.

[15] Major JM, Zhou EH, Wong HL, Trinidad JP, Pham TM, Mehta H, et al. Trends in rates of acetaminophen-related adverse events in the United States. Pharmacoepidemiol Drug Saf 2016;25:590-598. doi: 10.1002/pds.3906. 
[16] Tittarelli R, Pellegrini M, Scarpellini MG, Marinelli E, Bruti V, di Luca NM, et al. Hepatotoxicity of paracetamol and related fatalities. Eur Rev Med Pharmaco Sci 2017;21:95-101.

[17] Lee WM. Acetaminophen (APAP) hepatotoxicity-Isn't it time for APAP to go away? J Hepatol 2017;67:1324-1331. doi: 10.1016/j.jhep.2017.07.005.

[18] Ayonrinde OT, Phelps GJ, Hurley JC, Ayonrinde OA. Paracetamol overdose and hepatotoxicity at a regional Australian hospital: a 4-year experience. Intern Med J 2005;35:655-660. doi: 10.1111/j.1445-5994.2005.00947.x.

[19] Chan TY, Chan AY, Critchley JA. Paracetamol poisoning and hepatotoxicity in Chinese-the Prince of Wales Hospital (Hong Kong) experience. Singapore Med J 1993;34:299-302.

[20] Lee WM. Drug-induced hepatotoxicity. N Engl ] Med 2003;349:474-485. doi: 10.1056/NEJMra021844.

[21] Schmidt LE, Dalhoff K, Poulsen HE. Acute versus chronic alcohol consumption in acetaminophen-induced hepatotoxicity. Hepatology 2002;35:876882. doi: 10.1053/jhep.2002.32148.
[22] Kuffner EK, Dart RC, Bogdan GM, Hill RE, Casper E, Darton L. Effect of maximal daily doses of acetaminophen on the liver of alcoholic patients: a randomized, double-blind, placebo-controlled trial. Arch Intern Med 2001; 161:2247-2252. doi: 10.1001/archinte.161.18.2247.

[23] Chiew AL, Fountain JS, Graudins A, Isbister GK, Reith D, Buckley NA. Summary statement: new guidelines for the management of paracetamol poisoning in Australia and New Zealand. Med J Aust 2015;203:215-218. doi: $10.5694 / \mathrm{mja} 15.00614$.

[24] Teschke R, Schulze J, Eickhoff A, Danan G. Drug induced liver injury: Can biomarkers assist RUCAM in causality assessment? Int J Mol Sci 2017;18: 803. doi: 10.3390/ijms18040803.

[25] Teschke R, Zhu Y. Paracetamol (acetaminophen), alcohol and liver injury: Biomarkers, clinical issues, and experimental aspects. SL Pharmacology and Toxicology 2018;1:131.

[26] Danan G, Teschke R. RUCAM in drug and herb induced liver injury: The update. Int J Mol Sci 2015;17:14. doi: 10.3390/ijms17010014. 